\title{
The Origin of the Universe
}

\author{
JOÃO E. STEINER
}

$\mathrm{T}$

He ORIGIN Of THINGS has always been a central concern for humanity; the origin of the stones, the animals, the plants, the planets, the stars and we ourselves. Yet the most fundamental origin of them all would seem to be the origin of the universe as a whole - of everything that exists, without which there could be none of the creatures and things mentioned above, including ourselves.

Perhaps that is why the existence of the universe, its origin and nature, has been a subject of explanation in almost all civilizations and cultures. In fact, every culture known to anthropology has had a cosmogony - a history of how the world began and continues, of how mankind was created and of what the gods expect of us. The understanding these civilizations had of the universe is very different to what science teaches us today. However, the absence of a cosmology in these societies, of some explanation for the world in which we live, would be just as unthinkable as the absence of language itself. These explanations, for want of other frameworks from which to approach the subject, always had religious, mythological or philosophical foundations. Only recently has science been able to give its version of the facts, chiefly because science is recent

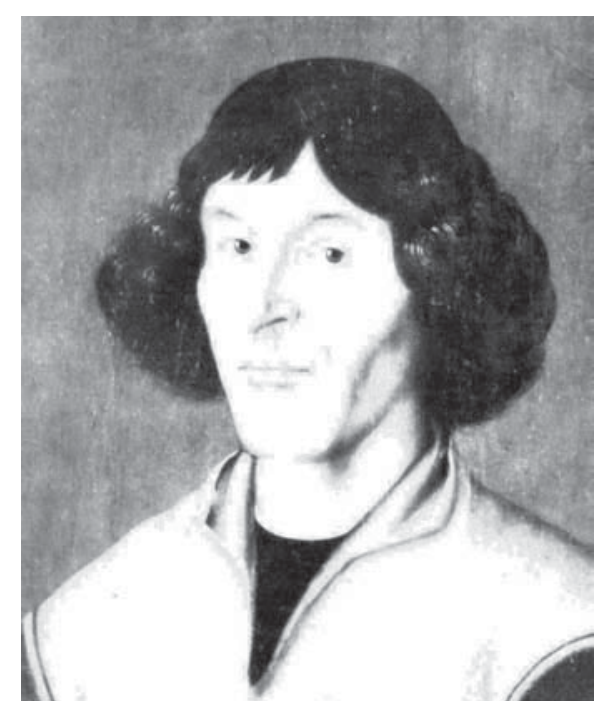
Nicolaus Copernicus (1473-1543). itself. In terms of experimental scientific method, Galileo Galilei (1564-1642, Italian astronomer, physicist and mathematician) is something of a milestone, though the Greeks had already developed sophisticated geometrical methods for measuring the orbits and sizes of celestial bodies and for predicting astronomical events. Nor can we forget that the Egyptians and Chinese, like the Incas, Mayas and Aztecs, also knew how to read the movements of the stars.

It is surprising how we can understand the physical universe in a rational manner and that it can be researched through the methods of physics and astronomy developed in our laboratories and observatories. The perception of this scientific dimension and capacity was revealed to us most clearly in the first, second and third decades of the $20^{\text {th }}$ Century. But the history of 


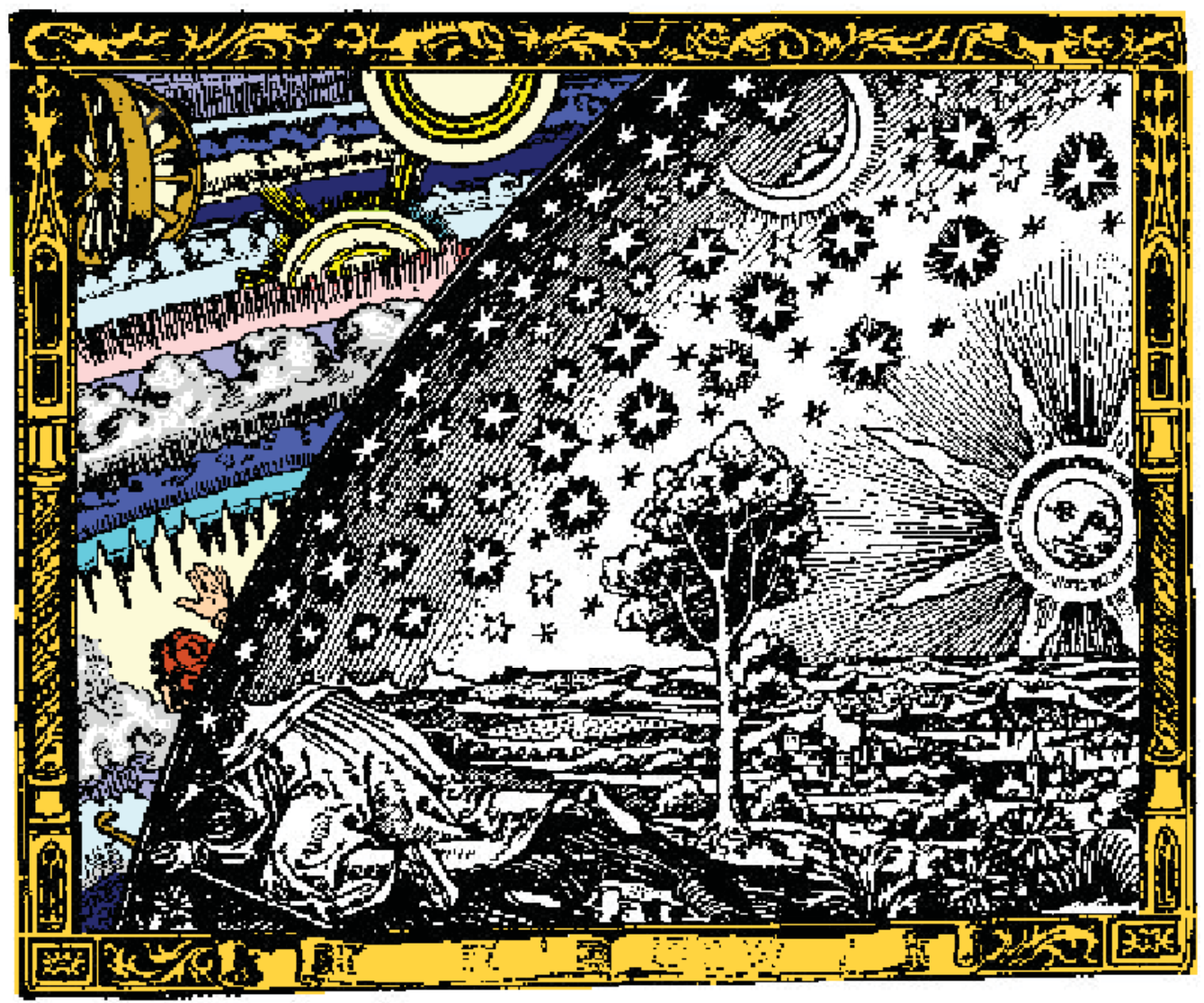

Figure 1 - The Flammarion woodcut (19 $9^{\text {th }}$ Century), illustrating the FlatEarth cosmology. Seen from the observer's village, the Earth seems flat, as encountered in everyday experience. However, just to the left, a "curious" fellow decides to breach the sphere of the fixed stars to sneak a peek at the mechanisms that move the Sun, Moon and planets.

cosmology (the structure of the universe) and cosmogony (the origins of the universe) neither begin nor end there.

\section{Flat-Earth Cosmologies}

So what was the cosmovision of the ancient Egyptians, Greeks, Chinese, Arabs, Incas, Mayas and Tupi-Guaranis? How did they, deprived as they were of modern astronomy, understand the universe? For almost all civilizations, it was necessary to fit in not only the visible face of the Earth and the sky above, but also the realm of the dead, both the blessed and forsaken, and the domains of the gods and demons. First of all, the world comes to us in everyday experience as if it were flat. Secondly, many cosmologies were interpretations of the physical or cultural reality of the civilizations in question. Thus, for example, the universe of the ancient Egyptians was a flat island divided by a river and covered over by an arched roof supported on four 
pillars. In ancient India, the various cosmologies of the Hindus, Brahmans, Buddhists, etc. shared a notion of reincarnation that the physical conditions of the world had to accommodate in all the various levels of heavens and hells that demanded. For the Hindus, for example, the universe was an egg encased in seven concentric shells, each wrought of a different element. The Babylonians, on the other hand, imagined a universe in two tiers joined by a cosmic staircase. The Mayan civilization was heavily dependent on corn and the often sparse rains that fell from the sky. So, for the Mayas, in the beginning the creator was alone with the sky and the sea, until, after various failed attempts, he finally managed to create people from corn and water.

In the Judeo-Christian Old Testament, the Earth was canopied by a mysterious firmament dividing the waters, the gates of the Abyss, Limbo and the House of the Winds. The Book of Genesis also tells us that the world had a beginning: "In the beginning God created the heavens and the Earth. The Earth, however, was shapeless and void; the darkness was upon the face of the deep and the Spirit of God hovered above the waters. God said, 'Let there be light'. And there was light. God saw that the light was good and separated darkness from light. God called the light DAY and the darkness NIGHT. There was evening and there was morning, one day".

\section{Geocentric Models}

Roughly 2,400 years ago, the Greeks had already devised sophisticated geometric methods and systems of philosophical thought. It should be no surprise, therefore, that they also proposed a more sophisticated cosmology than that of the flat universe. Theirs was in fact spherical, with the Earth ringed by celestial bodies that kept predictable orbits, and all encased by a fixed starry sky. An initial version of the geocentric model was presented by Eudoxus of Cnidus (c.400 - 350 BC, a Greek astronomer and mathematician born in present-day Turkey), and was followed by successive modifications. One of its revisions was proposed by Aristotle (384-322 BC), who demonstrated that the Earth was a sphere. He arrived at this conclusion after observing the shadow cast during a lunar eclipse. He also calculated the size of the Earth - at 50\% larger than it really is. Aristotle's geocentric model consisted of 49 concentric spheres which he believed could account for the movements of all of the celestial bodies. The most external sphere was that of the fixed stars, which controlled the behaviour of the inner spheres. The starry sphere, in turn, was controlled by a supernatural mover (entity).

The Greek geocentric model underwent further revisions. Eratosthenes (276-194 BC, Greek writer, born in present-day Libya) used an experimental method to measure the circumference of the Earth, which he overestimated by only $15 \%$. Ptolemy (Claudius Ptolomeus, $2^{\text {nd }}$ Century BC, Egyptian astronomer and geographer) revised Aristotle's model by introducing epicycles, a model in which the planets swivel in smaller circles as they orbit the Earth. 


\section{The Heliocentric Model}

The idea that the Sun is at the centre of the universe and that the Earth revolves around it, known as the heliocentric theory, was first proposed by Aristarchus of Samos (320 -350 BC, Greek mathematician and astronomer), who arrived at the notion based on his estimates of the sizes and distances of the Sun and the Moon. He concluded that the Earth revolves around the Sun and that the stars compose a fixed and very distant sphere. His theory attracted little attention, mainly because it contradicted the geocentric theory of Aristotle, then held in the highest prestige, and because the very idea of the Earth moving about was not particularly appealing.

About two thousand years later, in 1510, Copernicus (Nicolaus Copernicus, 1473 -1543, Polish astronomer) set down his own heliocentric model in the work Commentariolus, which circulated anonymously; Copernicus seemed to have foreseen the furore the theory would provoke and only allowed it to be published after his death. The work was brought out openly for the first time in 1543 under the title De Revolutionibus Orbium Coelesti, and carried a dedication to Pope Paul III.

The heliocentric model did not only trigger a revolution within astronomy, but also had enormous cultural impact, with philosophical and religious ramifications. The Aristotelian model was so deeply engrained in the psyche that plucking man from the centre of the universe proved an extremely traumatic experience.

In the end, Copernicus' heliocentric model won through as the correct cosmology, which raises the question of why Aristarchus' model had not managed to do the same 2,000 years earlier. The basic reason is that heliocentricity did not offer any real differential at the time when compared to the geocentric model. The measurements were a little fuzzy and one theory served just as well as the other in practical terms. Indeed, the geocentric model seemed to fit better with everyday reality, and it had the added bonus of being a homocentric model, which sat more comfortably with the philosophical and theological schools.

After the publication of Copernicus' theory, however, certain technological and scientific advances rendered it clearly superior to the Ptolemaic system. Tycho Brahe (1546-1601, a Danish astronomer) played an important role in advancing instrument-based techniques for making precise measurements with the naked eye, as refracting glasses and telescopes had not yet been invented. These measurements were roughly ten times more precise than earlier calculations. In 1597 Brahe moved to Prague, where he hired Johannes Kepler (1571-1630, German mathematician and astronomer) as his assistant. Later, Kepler was to use Tycho's measurements to establish his laws of planetary motion. These laws showed that the planets move in elliptical orbits with the Sun at one focus. With this realization, theoretical calculations and measurements acquired much greater congruity than under the older system. 


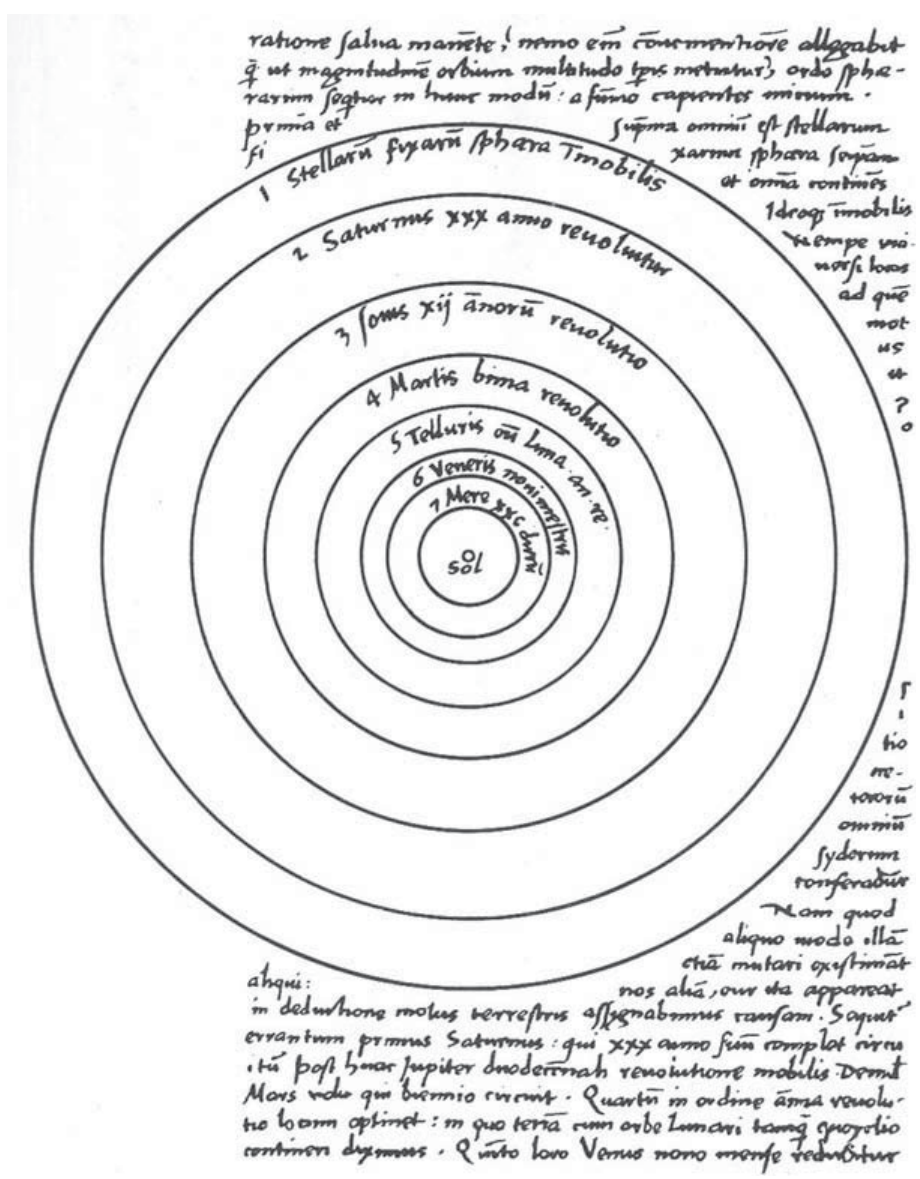

Figure 2 - This diagram from Copernicus' original manuscript places the Sun at the centre of the universe.

If for no other reason, the precision and the economy this afforded would have proved so important on long sea voyages that the laws would have imposed themselves for practical reasons alone.

In developing the telescope, Galileo created an instrument of vital importance to astronomical research, as it lends extraordinary powers of magnification to the human eye. When he trained his telescope at the Sun, he discovered sunspots; when he focused on Jupiter, he discovered its first four moons; turning to the Milky Way, he revealed that it was composed of myriad stars.

\section{The discovery of the galaxy}

It was precisely with the development of optical, mechanical and photographic techniques that the distances of the nearest stars could finally be determined, thus dispelling the notion of a sphere of fixed stars. With stellar distances now measured - and understood to be incredibly long - the interpretation gradually began to take hold that the stars and the Sun were 
objects of the same nature. Each star therefore had the "right", in principle, to host a planetary system.

One of the first consistent conceptions of the nature of the galaxy - and surprisingly accurate at that - was made by Kant (Immanuel Kant, German philosopher, 1724-1808), who, at the age of only 26 and thus long before he was to make his name in philosophy, came into contact with Newtonian thought and developed the idea that the solar system had originated from the condensing of a gas disk. He also formulated the notion that the solar system belongs to a much larger, compressed structure - what we call a "galaxy" today - and that the many nebulae then observed as diffuse stains were in fact similar systems, which he called "island universes".

The most important observational advances in terms of a more detailed understanding of the distribution of the stars were made by Wilhelm Herschel (1738-1822, German-born English astronomer and musician), the first to build large telescopes capable of viewing the fainter objects of the Heavens with more precision.

Stars are both scattered throughout space and grouped into so-called clusters. Studying these clusters, Herschel found that the stars were not randomly distributed, but that they followed a certain configuration (which we now call galaxies) discernable to the naked eye, just like the Milky Way.

The Sun, the star closest to the Earth, is some 159 million kilometres away, or rather eight light-minutes, which is the time it takes sunrays to travel from the King-Star to the surface of the Earth. Mapping the globular star clusters revealed that the galaxy is approximately 90 thousand light-years in diameter and made up of roughly 100 billion stars, all revolving around the same nucleus, some 25 thousand light-years from the Sun. It soon became clear that there are innumerable similar formations in the universe - the Nebulae, generically referred to today as galaxies.

When we look at the closest star outside our solar system, Alpha Centurion, we are really looking into the past. This star is 4.3 light-years away, which means that the light arriving here today was emitted 4.3 years ago. What we see, in fact, is the past. When we look at our neighbouring galaxy, Andromeda, we are really seeing it as it was 2.4 million years ago. Many of the stars we can observe today ceased to exist eons ago.

\section{The Big Bang theory}

In the 1920s, the American astronomer Edwin Hubble sought to establish a relationship between the distance of a galaxy and the speed at which it is either approaching or moving away from our own. The speed of a galaxy can be clocked with relative ease, but the distance requires a whole chain of tasks that makes it laborious and relatively imprecise work. After painstaking research, Hubble identified a correlation between the distance and the speed of the galaxies he was studying. The more distant the galaxy, the greater its 


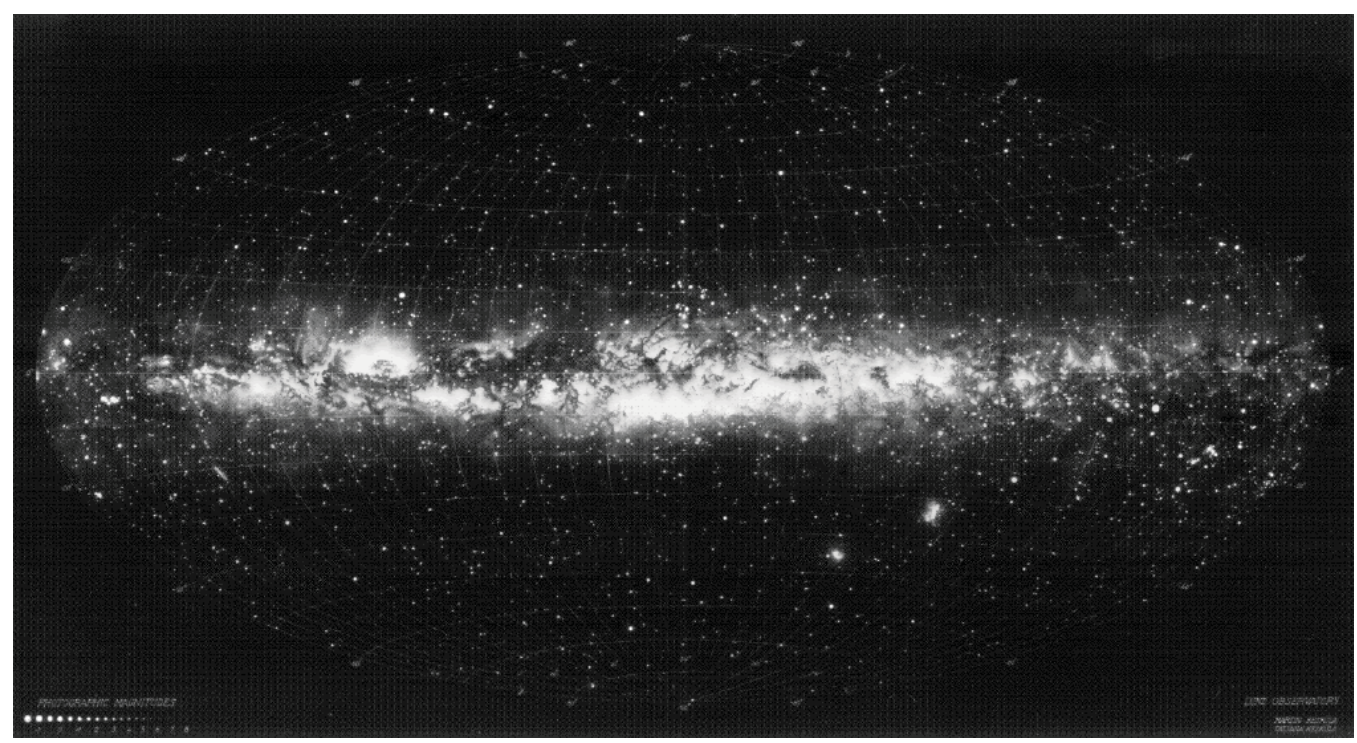

Figure 3 - a mapa mundi of the sky obtained by pasting together a series of photos as a planisphere. Here the Milky Way can be clearly seen. The white band is a composite of the light from 100 billion stars. The dark patches do not represent the absence of stars, rather they are dense concentrations of gas and dust blocking out the stars behind them. These clouds tend to condense to form stellar nurseries.

recession velocity. Basically, galaxies near to ours recede more slowly, while galaxies farther away, withdraw more quickly. How can we explain this law?

At first, one might be tempted to think that this restores us to the centre of the universe, that ours is a privileged position after all. All the other galaxies know we are here and for some reason are moving away from us. This explanation is, of course, hardly Copernican. At this stage in the game no-one actually believed anymore in the cosmic centrality of mankind, so there had to be another explanation.

This other explanation can be easily understood if we make a twodimensional analogy for the universe. We tend to think of our universe in terms of three-dimensional space; we can walk forwards, sideways and even jump up and down. To these we can add a further dimension: time. These four dimensions constitute the spacetime universe in which we live, but we could imagine other universes. From a mathematical perspective, we can, for example, imagine various two-dimensional universes; the surface of a ball is a two-dimensional entity, as is the surface of a table. Imagine the surface of a child's balloon as a two-dimensional universe. We can draw two-dimensional galaxies on that surface, populated by two-dimensional ants. Some of these ants might be astronomers whose task it is to observe the other galaxies and measure their distances and speeds. 


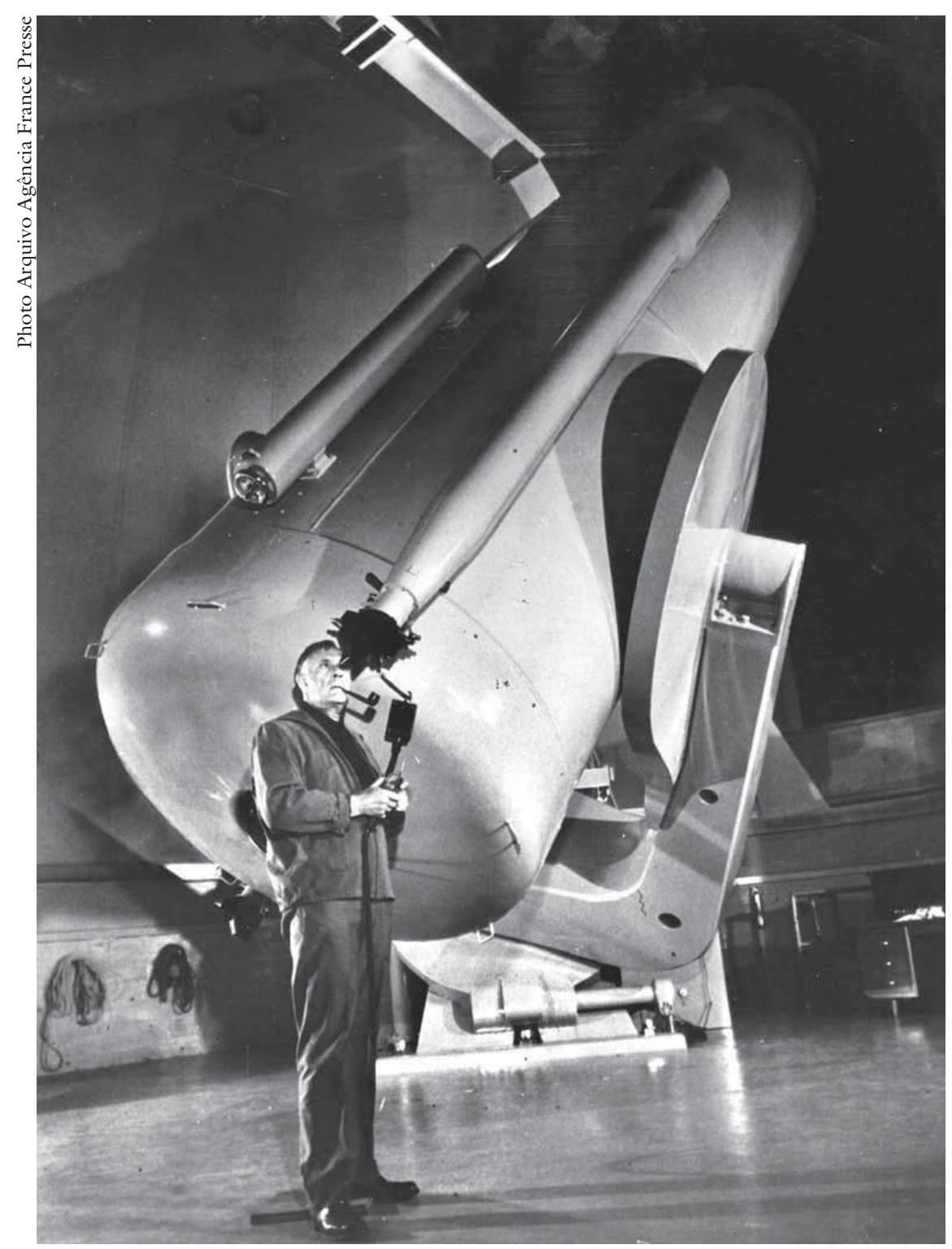

Edwin Hubble (1889-1953) at the Schmidt telescope on Palomar Mountain, California, in 1949.

Let's imagine for an instant that someone blows into the balloon and makes it expand. What will the astronomer-ant see? Basically, he will see that the galaxies closest to him recede slowly while those more distant shuttle away at a faster speed. This ant will have discovered Hubble's Law. If we imagine the opposite - that instead of expanding, the balloon begins to deflate-, what the ant will see is all of the galaxies edging closer to each other - the opposite of Hubble's Law. What the law proves, therefore, is that our universe is in expansion! In other words, it will be larger in the future and it was smaller in the past. The further back in the past, the smaller the universe. If we follow the logic through, we can imagine a balloon so small that it shrinks to a mere 
pinhead. From this initial pinhead we need not stretch the imagination to arrive at the idea that the universe started with an explosion - the so-called Big Bang - and that it has been expanding ever since, as Hubble's Law confirms. So how long ago did this happen? The most recent estimates put the Big Bang at $13.7( \pm 0.2)$ billion years ago. ${ }^{1}$

Indeed, theoretical work from 1927 by the Belgian abbot Georges Lemaitre shows that Albert Einstein's General Theory of Relativity is actually compatible with the recession of the nebulae (as galaxies were called back then) and he was the first to suggest that the universe had originated from an explosion, from a "primeval atom".

One question that may spring to mind is to which corner of space we should be looking in order to see where this explosion occurred. If the universe is expanding, what is it expanding in? Well, in our balloon model - a two-dimensional universe - the Big Bang occurred at the centre of the balloon, not on its surface. Space is the surface. The interior is the past, and the exterior, the future. The centre is the origin of time. So the explosion

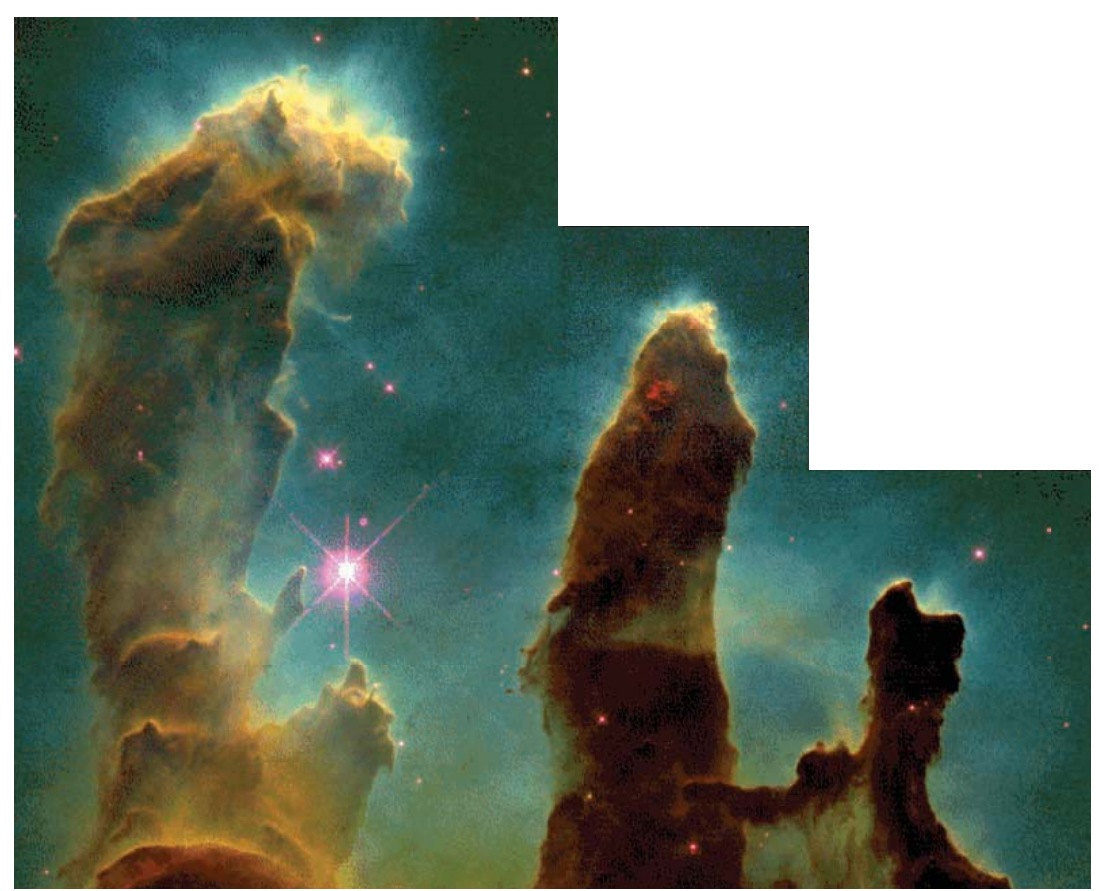

Figure 4 - M16 Nebula, image obtained by the Hubble space telescope (courtesy of NASA). This nebula is basically a nursery where new stars are being born. The light from the fledgling stars shines through the gas pillars in which they are forming.

did not occur in space, but at the beginning of time, and space itself is the product of this temporal singularity. This simple example shows us how the two-dimensional model is an intuitive, but reliable illustration of the 
fundamental issues of cosmology; adding a third dimension is just a question of mathematical skill!

Let us return to the notion that to look far into the distance is to look back into time. Could we actually see the universe evolve? The idea seems interesting; the deeper we look into space, the younger the universe we encounter. Could we then observe the moment the galaxies were born? Yes, if we had the technology. All we need is instruments that allow us to look back through 12 billion light-years of space. This technology is already available in the newer, larger telescopes. These instruments can enable us to see when, how and why the galaxies were born - and this is one of the most thrilling areas of contemporary science.

Another question that naturally arises is: when was time-zero and what was there before it? The theory of relativity deduces that the density at timezero must have been infinite. To tackle this situation would require a quantum theory of gravitation that does not yet exist, and so we cannot offer a scientific treatment of it at this time. Understanding this phase in the history of the universe is one of the greatest unsolved problems in contemporary physics.

\section{Confirmations of the Big Bang}

In the late 1940s, the astronomer George Gamow suggested that the initial explosion may have left some still observable traces. His reckoning was that a universe so hot and dense would have emitted a lot of light. With expansion, the characteristic temperature of this light would have dropped. According to simple calculations, perhaps it was still observable today in microwave radiation, with a temperature of some 5 kelvins. In 1965, two engineers, Arno Penzias and Robert Wilson, were trying to find the source of an electromagnetic noise that was disrupting the reception of a transmissions system they were testing. They discovered that the static continued no matter where they pointed the antenna. When they measured the radiation, they found a value close to that expected for cosmic background radiation, 2.7 kelvins (close to absolute zero). It was confirmation of the Big Bang theory and the discovery earned Penzias and Wilson the Nobel Prize for Physics in 1978.

In science, whenever you make a prediction based on a given theory and that prediction is confirmed, it strengthens the theory. This was exactly what happened with cosmic background radiation. It was a point for the Big Bang, which now achieved total supremacy

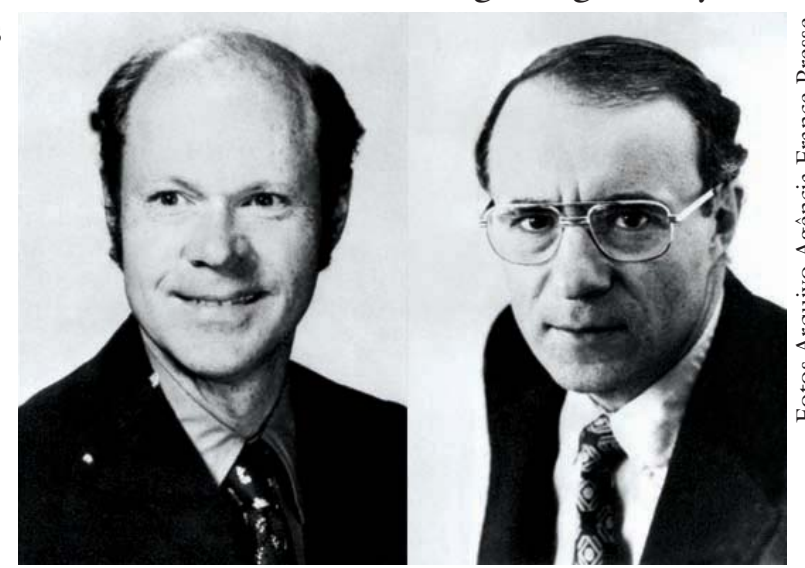

Robert Woodrow Wilson and Arno Penzias. 


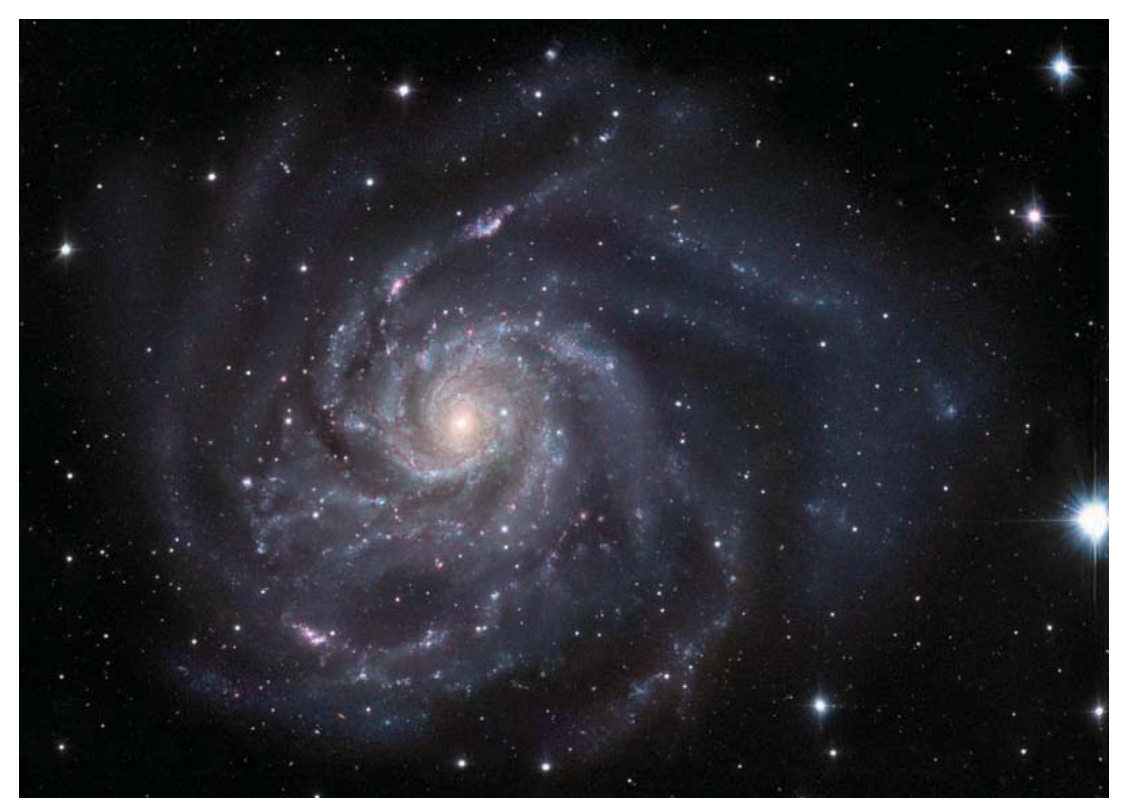

Figure 5 - A spiral galaxy similar to the one in which we live. Each galaxy like this is roughly 100 thousand light-years in diameter and contains around 100 billion stars. The brighter patches around the arms of the spiral are stellar nurseries. The denser stars are blue and die more quickly, while the lighter stars are red and burn longer.

over its steady state rival, according to which the universe is the same today as it has always been.

This was not the only confirmation of the theory. Big Bang theorists also expect that the element helium would have formed within three minutes of the explosion and that a quarter of the material in the universe resulted in this element, with the other three-quarters forming hydrogen. When science finally managed to measure the primeval abundance of helium, the value was exactly as predicted. ${ }^{2}$

\section{Inflation, dark matter and dark energy}

Despite the observational evidence in favour of the Big Bang, the viability of the theory remained a bone of contention for quite some time. Various theoretical difficulties hindered the precise description of the observations until, in 1982, the American physicist Alan Guth proposed a solution that many initially regarded as flight of fancy: the Inflationary Big Bang theory. The basic idea behind this theory is that universe underwent a phase of extraordinary expansion. When the universe was only a trillionth of a trillionth of a trillionth of a second old (sic), the universe expanded exponentially (by a factor of 1 followed by a sequence of 50 zeros!). This model dispenses with a number of theoretical problems. Naturally, as one would expect, such a fanciful proposal initially lacked concrete 
evidence capable of grounding it in reality, but it did not take long for such evidence to appear. Inflation theory argues that the universe derived from seeds generated during the inflationary period by quantum fluctuations corresponding to Heisenberg's Uncertainty Principle, only multiplied by the factor of inflation. The fact that it implied the existence of small fluctuations in the temperature of cosmic background radiation meant that the theory was testable. A lot of research was conducted until, in 1992, the Cobe satellite not only proved that such fluctuations exist, but that they behave exactly as predicted by inflation theory. This research earned the NorthAmerican researchers George Smoot and John Mather the Nobel Prize for Physics in 2006.

So what caused this inflation? The consensus is that it must have been a phase transition, much like occurs when water turns to ice. When water freezes to ice it releases its latent energy. A phase transition occurring at the moment of the Big Bang would likewise have unleashed latent energy, thus causing rapid and sudden expansion in the nascent universe.

Galaxies are often found in agglomerations, called clusters. The Virgo cluster contains eight hundred galaxies, while the Coma holds two thousand. In 1933, the Swiss astronomer Fritz Zwicky measured the mass of a galaxy cluster and found that it was 400 times heavier than the sum of its individual galaxies. This meant there was "dark matter" underpinning the agglomeration and keeping its stability. This research languished in discredit for many decades until new measurements made of other galaxies in the 1960s also pointed to the existence of this mysterious dark matter. In the Milk Way, dark matter outweighs visible matter in the form of stars and gas by ten to one. Wherever you look in the universe, this mysterious matter seems to be there. But what exactly is it? There has been a great deal of speculation as to its nature, but a convincing answer is yet to be found. All we do know is that its behaviour is very different from that of normal matter.

What, then, is the outlook for the future expansion of the universe? That depends on its mass content. If this is very high, the universe will eventually slow to a halt, whereupon it will probably begin to shrink. This is known as the closed universe model. If the mass is low, it will not be enough to slow it down and the universe will go on expanding forever. This is called the open universe model. The threshold between the two is the flat universe ${ }^{3}$.

Attempts to determine which model corresponded to the reality drove a great deal of research over the decades. In 1998, at the close of the century and the millennium, it was discovered that the universe is not slowing down, but actually speeding up. In other words, the more time that passes, the faster the galaxies pull away from each other. This was a fascinating and disquieting discovery, as it suggested the existence of an energy that works against gravity, so-called "dark energy". Dark energy has no connection with dark matter, as 


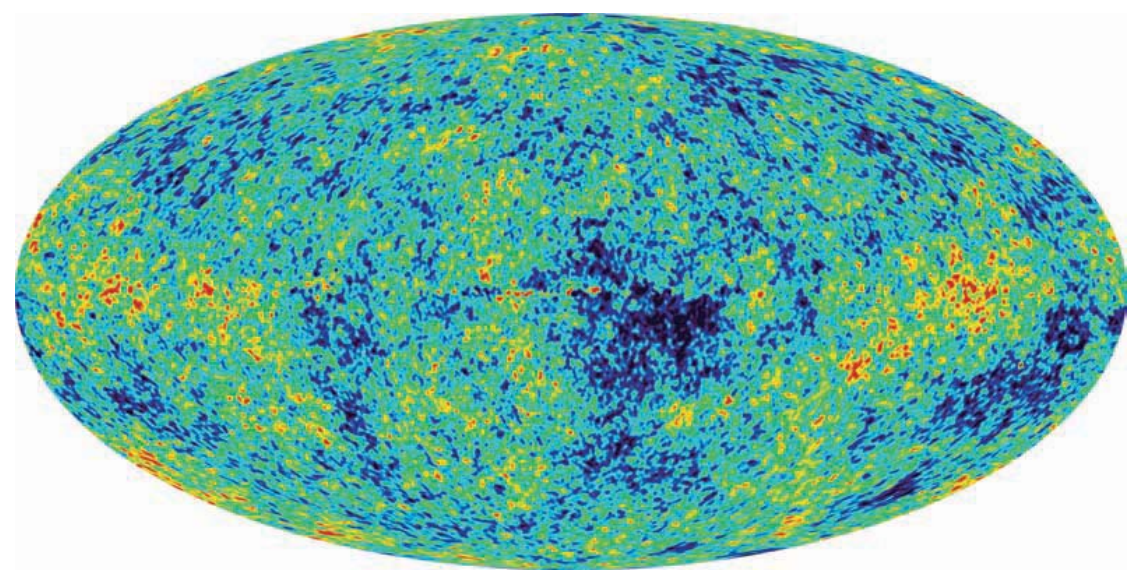

Figure 6 - "Mapa mundi of the Microwave Sky" obtained by NASA's WMAP satellite and showing cosmic background radiation structures. These fluctuations are miniscule and equivalent to one part per one hundred thousand on the temperature scale. These fluctuations prove the inflation theory, which claims that the universe expanded exponentially in the first trillionth of a second after the Big Bang, triggering an incalculable number of quantum events in the process, which left miniscule fluctuations pervading recently-created spacetime on all scales. The map is a photograph of the universe when it was only around 300 thousand years old. Later on, some of the fluctuations shown above collapsed to form galaxies, galaxy clusters and even larger structures.

the latter possesses gravity whilst the former does not. Quite the contrary, in fact - it repels.

The most recent measurements indicate that the mass-energy of the universe consists of $4 \%$ normal matter, $22 \%$ dark matter and $74 \%$ dark energy. As we only know normal matter, we are therefore oblivious to $96 \%$ of the universe. In other words, we know only the tip of the iceberg. ${ }^{4}$

\section{Beyond...}

The more we research, the further back we push the frontier of natural knowledge. As technology advances, our measurements and information become more precise, and our theories, more sophisticated and detailed. But at the end of the day, if Newtonian mechanics (Isaac Newton, English physicist and mathematician, 1642-1727) seems to work so well in our daily lives, why do we need complex Quantum Mechanics and Relativity Theory? Because the technology has progressed and the more accurate measurements it now gives us can only be explained by such theories. Just as in physics, the dialogue between technological development and scientific advancement has always been present in the history of astronomy, and with fertile effect for both. 
We only believe in Quantum Mechanics because it works, not because it seems to make sense. That said, it is estimated that $50 \%$ of the global economy today is in some way connected with Quantum Mechanics. Without it, we would have none of the electronics that goes into computers, televisions, telephones, factory machinery, etc. The Cosmic Inflation Theory also works, though in a somewhat arcane way. It is useful insofar as it enables us to calculate the fundamental characteristics of the universe. So why not use it? After all, isn't that why seafarers adopted Copernican theory?

\section{Does the Big Bang explain everything?}

So is the Big Bang the definitive theory? Let us examine the hypothesis. All throughout history we can see that our concept of the universe has evolved. It has passed through various stages, all of which can be considered cosmological theories. The flat Earth, the geocentric model, heliocentricity, galacticocentricity, the Big Bang, the Inflationary Big Bang... Each model explains what was known at the time and what the measurements could confirm. We cannot say that these theories were wrong; perhaps it would be truer to say they were incomplete. After all, when it comes to everyday experience, the Flat-Earth model works rather well. But the Earth is round, and it revolves around the Sun, and so on, so forth. The discovery that the universe - the all - evolved in a manner that can be rationally analysed may strike us as surprising. More surprising still, however, is that we can even demonstrate that it had an origin. The laws that we have developed on our little planet apply to the entire universe. There is no evidence to suggest any measurable discrepancy.

Is that the end of the matter? Everything would suggest that the answer is no. if we are Copernicans in terms of space, we must also be Copernicans in relation to time, accepting, that is, that ours is no privileged, special moment. The Big Bang should the subject of rationalization, mined for detail. We have already finished Chapter One: the Big Bang did not just happen any old way, it was inflationary. How many more twists will there be in the human adventure to decipher the nature of the universe in which we live?

The concept of the universe in the $17^{\text {th }}$ century had already incorporated Newtonian notions of space and time. The universe seemed static and infinite, far removed from the mindset that so intimately intertwined the destinies of the gods and man with the concept of the world. The French philosopher Blaise Pascal expressed it thus: "Drawn into the infinite immensity of space, of which I know nothing and which knows nothing of me, I am terrified... the eternal silence of infinite space alarms me".

But are we quite as alone as Pascal feared? Are human nature and destiny so totally disconnected from the greater cosmic structure? Today we know that each star could hold a solar system and that each galaxy contains an average of 100 billion stars. It is therefore perfectly legitimate to suppose 
that the number of planets with conditions similar to those on Earth is immense, and that's just considering our galaxy. We must also remember that the number of galaxies observable on the accessible cosmic horizon is somewhere in the region of 100 billion. So it is clear that there could be an enormous number of planets with the right conditions to allow life to originate and flourish. That does not mean that human life like ours is a common occurrence, not least because it assumed its present form fortuitously, but also because it is certainly ephemeral on a cosmic timescale. Precisely because of this ephemeral nature and the distances involved, it is well nigh impossible for civilizations of a similar degree of development to establish contact, even if they did exist simultaneously on separate stars or indeed in separate galaxies.

Another connection that binds us to the stars is the set of chemical elements, indispensable to our physical structure. Every atom of oxygen we breathe, or of calcium in our bones or of iron or carbon in our musculature can be traced back to a very specific origin. Only hydrogen and helium (as well as deuterium and part of lithium) were formed by the Big Bang, the heavier chemical elements were all synthesized in the cores of stars and launched into space upon their deaths, where they blended with the remains of thousands of other stars to form a new generation of celestial bodies. The Sun is already a third-generation star, and it is thanks to this that the chemical composition of the solar system is rich enough to form life as we know it.

Scientific cosmology, unlike traditional cosmologies, does not attempt to link the history of the cosmos to how mankind ought to behave (as adepts of astrology still do today). It is the role of scientists, philosophers, artists and other creative individuals to understand it and express the human sense of it. The full impact of this cosmovision on human culture will only be truly known when our physical reality is plainly understood by the common citizen.

Until then, the role of astronomy is to tell us where we are, where we came from and where we are going. From the look of it, this mission would appear to have no end.

\section{Notes}

1 As a rival to the Big Bang theory, the Steady State theory persisted for many years. This theory was based on the Perfect Cosmological Principle, according to which the universe is homogeneous, isotropic and constant in time. This model was a favourite of physicists, particularly because it eliminated the problem of their having been an origin of time. This principle is incompatible with observations that the universe evolved over time.

2 There is other evidence to support the Big Bang theory. If the universe were not finite, the night sky would not be dark. Furthermore, galaxies evolve over time, i.e. they age, becoming laden with more heavy chemical elements. This is borne 
out by direct observation. The further we peer into the universe, the younger (less evolved) the galaxies are.

3 If the universe were closed, it would at some stage begin to shrink, resulting in a Big Crunch. This, in turn, might be followed by another Big Bang, and so on so forth, which would imply that the universe were cyclical - an idea shared by many ancient cosmologies. However, the Inflation Theory is incompatible with the idea of a closed universe.

4 Big Bang research inspired laboratory experimentation in physics that led to the discovery of a new kind of particle. Contrary to the tendency during the $20^{\text {th }}$ Century, when breakthroughs in physics were used to further our understanding of the universe, today, it is cosmology that sets the course for physics, indicating where it ought to look in order to better understand the material world.

\section{Bibliography}

DAMINELI, A. Hubble: a expansão do universo. São Paulo: Odysseus, 2003.

FERRIS, T. O despertar da Via-Láctea. Rio de Janeiro: Campus, 1990.

GLEISER, M. A dança do universo. São Paulo: Companhia das Letras, 2000.

GUTH, A. The Inflationary Universe. Massachusetts: Addison-Wesley, Reading, 1997.

KEPLER, S. O.; SARAIVA, M. F. Astronomia e astrofísica. Porto Alegre: Editora da UFRGS, 2000. [Also available at: astro.if.ufrgs.br]

NORTH, J. Astronomy and Cosmology. Glasgow: Fontana Press, 1994.

ABSTRACT - THE VARIOUS cosmological models along history are briefly reviewed. The evolution of the ideas may be understood as successive models such as the flat earth, the geocentric models as well as the heliocentric and the galacto-centric ones. In the last century, a concept was developed, the big-bang theory, that describes the most sophisticated observations on hand today and shows that the universe had an origin that can be search with scientific methods. In recent decades this model was refined to a new concept: inflation. By the end of the century and millenium new discoveries showed that all known matter is only the tip of the iceberg in a universe dominated by dark energy and dark matter whose natures remain mysterious.

KEYWORDS - Universe, Cosmology, History of Astronomy, Big Bang.

Joz̃o E. Steiner is the director of the Institute of Advanced Studies at the University of São Paulo and a professor at the Institute of Astronomy, Geophysics and Atmospheric Sciences at the same university.

Translated by Anthony Doyle. The original in Portuguese is available at http://www. scielo.br/scielo.php?script=sci_issuetoc\&pid=0103-401420060003\&lng=pt\&nrm=iso.

Received on 8.30.2006 and accepted on 9.11.2006. 